disk to disks which are held by all consultants and trainees, and to a central computerised database thus building up a bank or textdisk (cf textbook) of presentations. Although the prescribed format may appear restrictive, it is itself evidence based ${ }^{2}$ and it contributes to consistency and, in our experience, quality. Of course, the use of other additional teaching aids, for example flip charts, is not excluded. Trainees are obliged to attend except when on holiday or study leave. Audit of this has indicated $100 \%$ compliance. The programme has proved popular and has attracted the participation of two hospitals outside the region and of several non-career grade staff.

M F NICOL

KE JONES

M CAMERON

J KENDALI

Accident and Emergency Department, Musgrove Park Hospital, Taunton, Somerset TA1 5DA

1 Davies F, Geary U, Soulsby T, Good A. How we did it: the development of a specialist registrar training programme by the Mersey Accident and Emergency Trainees' Association. J Accid Emerg Med 1997;14:321-3.

2 How to do it, vols 1-3. London: BMJ Publishing Group.

\section{Developments in radiology}

EDITOR,-I read with interest the summary of current developments in radiology by Dr D Lloyd.' Our A\&E department has been using digital radiology (Kodak/Laserlines) since January 1996. At the time we were provided with state of the art PCs for viewing the images, but they were not sufficiently powerful and viewing a series of images could be slow. Each image can be $10 \mathrm{Mb}$ in size! In view of the speed penalty and the lack of provision of an archiving facility by Kodak, we have been using films with the facility of viewing the image on screen when necessary. So far the advantages are:

(1) Speeding up of the $x$ ray reporting system (radiologists can report from the digital image and don't have to wait for the $x$ ray films).

(2) The $x$ ray image can be left on the hard disk on the A\&E viewing stations for several days (if the hard disk is large enough). Therefore $x$ ray images are easily available if the patient returns, or if a wrong $x$ ray diagnosis is made they can be shown to junior staff.
(3) Compilation of a library of teaching $x$ ray images in TIFF format. These are captured using the clipboard facility on Windows NT and are usually between $500 \mathrm{~K}$ and $1 \mathrm{Mb}$ in size.

(4) Viewing of $x$ ray films digitised at a minor injury unit sent down an ISDN line or hospital network (to be implemented March 1998).

On an optimistic note, the technology appears to have progressed sufficiently to make the system workable in $\mathrm{A} \& \mathrm{E}$ with no speed penalty. However the minimum specification is a Pentium 2 MMX $233 \mathrm{MHz}, 128 \mathrm{RAM}, 4$ $\mathrm{Gb}$ wide SCSI hard drive, fast video card with 4 RAM, and 17 inch monitor. As an ideal, an $\mathrm{A} \& \mathrm{E}$ department should have as many viewing stations as they have doctors on duty.

The system however will not become cost-effective until Kodak provide an archiving facility, preferably using DVD (increased storage medium) CD ROM technology. Until then $x$ ray films will have to be produced in addition to the digital images.

RAY MCGLONE STUART DURHAM Royal Lancaster Infirmary, Ashton Rd, Lancaster LA1 4RP; email: Ray.McGlone@LAHT.NWEST.NHS.UK

1 Lloyd D. The value of current developments in radiology to the accident and emergency department - a pictorial review. J Accid Emerg

\section{BOOK REVIEW}

\section{Advanced Paediatric Life Support: The} Practical Approach, 2nd ed. By the Advanced Life Support Group. (Pp 306; £22.95.) London: BMJ Publishing Group, 1997. ISBN 0727910698

It is three years since the first edition was published and it has obviously been exceptionally successful from the fact that it has had three reprints before the second edition was published.

I think it is particularly impressive that the Advanced Life Support Group allows you to buy the APLS manual without having to attend the course. I am sure that this practice should be more widespread. Another commendable activity of the ALS Group is the fact that they invite all APLS instructors and providers for a "constructive critique" of the first edition.

There is no doubt that the working group have made significant changes to the first edition. Most notably they have tried to encapsulate the structured approach throughout the manual.

Included in the second edition are sections on diabetic ketoacidosis, the management of pain relief in children, and the structured approach to the seriously ill child. Another new section is on paediatric radiology which encapsulates the ABCS approach to interpretation of cervical spine, chest, and pelvic $x$ rays. The only deficiency is the lack of radiographs or line diagrams.

The working party has attempted to revise some of the algorithms or incorporate new ones, in line with national recommendations. This I believe is essential for consistency in respect of treatment algorithms. There is an increasing move towards evidence based medicine and I would prefer to see all the APLS protocols nationally recommended.

The section on the management of poisoning has also been altered to follow a structured approach. Some of this section is not entirely in keeping with the recommendations of the National Poisons Information Centres. There is very little mention of alcohol, carbon monoxide/smoke inhalation, solvents, and benzodiazepine overdose.

I would like to see more detail on meningococcal disease and triage (to include "Baby Check"), and more emphasis on the team approach. I also felt it was a pity that the paediatric resuscitation chart was not included.

The practical procedures section is very useful, and includes femoral venous access. A little more detail would be helpful: for example, intraosseous infusion should include details of contraindications and complications of this procedure.

Overall the APLS Manual will certainly achieve its aim to improve the emergency care of children.

RUPERT J EVANS Cardiff 